\title{
Wavelength Tunable Injection Locking of High Power Super Luminescent Diode with 1.4Watt Diffraction-Limited Output
}

\author{
${ }^{\mathrm{a}}$ Yi-Shin Su, ${ }^{\mathrm{a}}$ Chih-Hung Tsai and ${ }^{\mathrm{a}, \mathrm{b}}$ Ching-Fuh Lin \\ a with Graduate Inst. of Electron-Optical Eng., National Taiwan Univ., Taiwan \\ ${ }^{b}$ with Dept. of Electrical Eng.and Graduate Inst. of Electronics Eng., National Taiwan Univ., \\ Taiwan
}

\begin{abstract}
A new-type tunable high power wavelength tunable ASE light source is fabricated. The device length is $1960 \mu \mathrm{m}$. The waveguide is $100 \mu \mathrm{m}$ wide and tilted at $6^{\circ}$ from the facet normal. Without external feedback, this device can provide 1.4Watt ASE light per facet at 12Amp injection current. The emitted spectrum has 20nm FWHM. The far field has narrow divergence angle and the near field is smooth without filamentation. When external feedback is applied, the slope efficiency is increased from $0.15 \mathrm{~W} / \mathrm{Amp}$ to $0.2 \mathrm{~W} / \mathrm{Amp}$. Only 6Amp injection current is required to reach $1.06 \mathrm{Watt}$ output. Feedback from a grating can be used to control the emission wavelength and improve the beam quality.
\end{abstract}

Keywords: laser diode, broad area waveguide, ASE light sources, wavelength tunable light

sources

\section{INTRODUCTION}

The compact size, reliability and efficiency of semiconductor light sources make them ideally suitable for a wide variety of applications requiring high power levels. In applications such as frequency doubling ${ }^{1}$, free space communication $^{2}$, and material processing ${ }^{3}$, additional requirement on good laser beam quality is needed to achieve high brightness. In order to replace those power consuming solid-state lasers, high-power spatially coherent semiconductor light sources have been a goal of researches for years. The output powers of single-stripe devices are generally limited to a few hundred milliwatts.

To make a diode light source with thousands of milliwatts optical power, the emitting facet must be large to prevent catastrophic optical damage (COD). Even with a high COD threshold $\left(10 \mathrm{MW} / \mathrm{cm}^{2}\right)^{4}$, the emitting facet is required to be large to make COD threshold three times higher than the optical intensity for 1 watt operation.

Thus, more than $30 \mu \mathrm{m}^{2}$ broad emitting facets are necessary for watt-range operation ${ }^{5}$. However, the broad-area waveguide leads to the small difference of modal gain between the high-order modes and the fundamental mode. In addition, the nonlinear interaction between carriers and light will produce filamentation of optical power distribution $^{6}$. Thus, high-order transverse modes usually dominate the emitted light power of these devices and the spatial coherence of emissions on every part of the facets is poor. It is necessary to resolve the filamentation problem and design new types of semiconductor light sources that can provide high power and good beam quality.

Tapered waveguide power amplifiers ${ }^{7}$ have been used to maintain the beam quality with increased output power and emitting facet area. Although tapered waveguide system can provide watt-range output power, beam filamentation remains to be a problem ${ }^{8}$. A new type of broad area super luminescence light source has been reported to be capable of emitting 1.4 watt output with diffraction limited far field divergence ${ }^{9}$. Without any AR coating, the full width at the half maximum (FWHM) of the emitted spectrum of this device is $20 \mathrm{~nm}$. The spectrum is free of the modulation caused by Fabry Perot modes. Here we report that the emission wavelength of the similar device can be controlled by applying external feedback.

High-Power Diode Laser Technology and Applications IV, edited by

Mark S. Zediker, Proc. of SPIE Vol. 6104, 610411, (2006) · 0277-786X/06/\$15 - doi: 10.1117/12.645032

Proc. of SPIE Vol. 6104 610411-1 


\section{DEVICE STRUCTURE}

The laser diodes are fabricated on the wafer with multiple quantum wells (MQWs) designed for the wavelength at $1300 \mathrm{~nm}$. Figure 1 shows the waveguide configuration of this device. The waveguide pattern is modified from tilted broad area device. The tilted angle is $6^{\circ}$ from the facet normal. The emitting aperture of this device is $100 \mu \mathrm{m}$ in width. The total device length is $1960 \mu \mathrm{m}$. The dashed lines in figure 1 indicate the boundary of waveguide before modification. As shown in the figure, two triangular parts are removed from the waveguide. Figure 2 is the cross-section view of the device. The waveguide is formed by etched channels along the waveguide edge. The channels are etched by reactive ion etch with $\mathrm{CH}_{4}$ and $\mathrm{H}_{2}$ process gas. The bottom of channels is controlled to be $100 \mathrm{~nm} \sim 200 \mathrm{~nm}$ above the active layer. $300 \mathrm{~nm}$ of the Silicon oxide deposited by plasma enhanced chemical vapor deposition is used as passivation dielectric. RIE is used to etch the current conducting window. The topside electrode is Ti/Pt/Au. The bottom side electrode is $\mathrm{AuGe} / \mathrm{Ni} / \mathrm{Au}$.

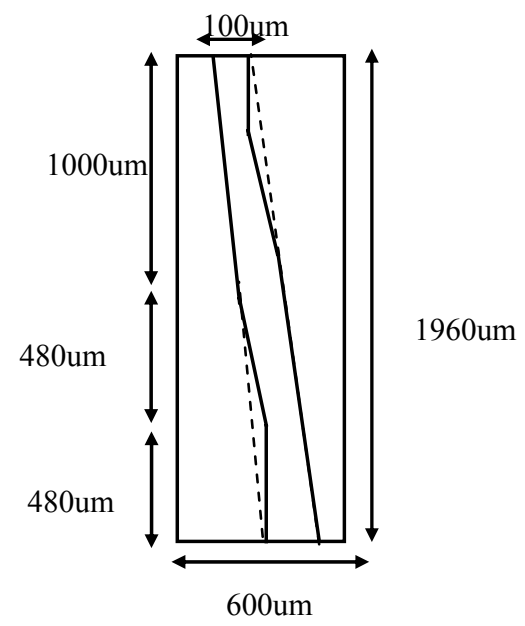

Figure. 1 Waveguide Design

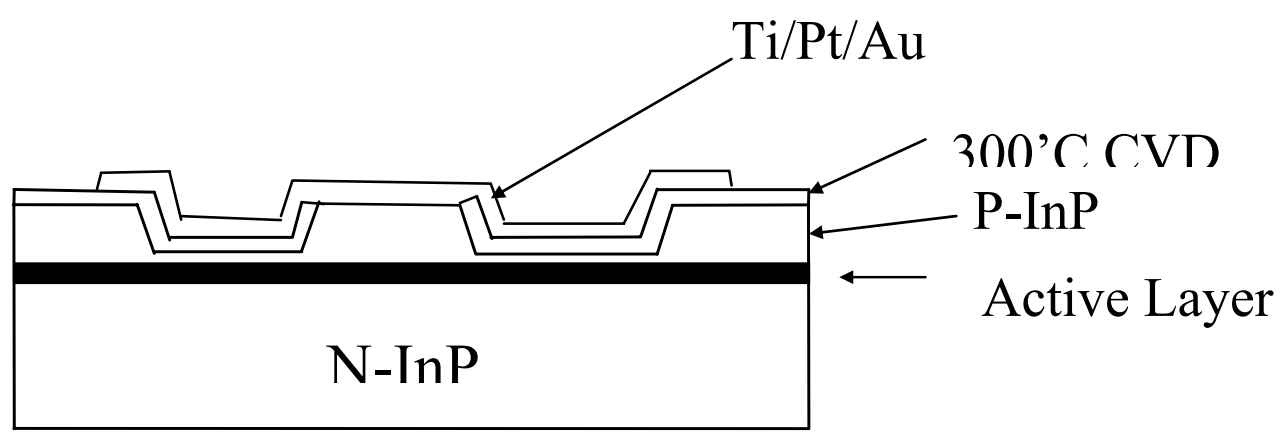

Figure 2 Device cross-section view. 


\section{DEVICE PEFORMANCE}

Without external feedback, the L-I curve of this diode has a threshold-like behavior at $1 \mathrm{Amp}$. Only $3 \mathrm{~mW}$ output power is observed below the threshold. Above the threshold current, the output power increases linearly with current until 6 Amp. The slope efficiency is $0.15 \mathrm{~W} / \mathrm{A}$. The maximum measured output power of this device is $1.4 \mathrm{~W}$ per facet when the current is $12 \mathrm{Amp}$. However, the overall emission spectrum is pretty broad. The measured spectrum has 20nm FWHM at 6Amp of the injection current, as shown in Fig. 4. The spectrum shows no obvious Fabry-Perot modes This device behaves more like a super luminescence diode.

Figure 5 shows the far field pattern of this device measured at $6 \mathrm{Amp}$. The far field has a sharp peak located at $32^{\circ}$ away from the direction of facet normal. The far field distributed over a pretty narrow angle. The FWHM is only about $2^{\circ}$. When the device is operated below threshold, the sharp peak at $32^{\circ}$ away from the facet normal is absent. When the device is operated above threshold, the light emitted from the device concentrates at $32^{\circ}$ away from the direction of facet normal. Because the emitted far field is at an angle from the facet normal, the device is unlikely to have oscillation between the cleaved facets. This probably explains why no obvious Farby-Perot modes are observed in the spectrum and the overall spectrum is broad. Figure 6 shows the near-field pattern of the device at $6 \mathrm{Amp}$. The measured near field pattern has $43 \mu \mathrm{m}$ FWHM. The near field mainly distributes within the waveguide. No filamentation is observed. From the measured near field and far field patterns, they correspond to the diffraction limit.

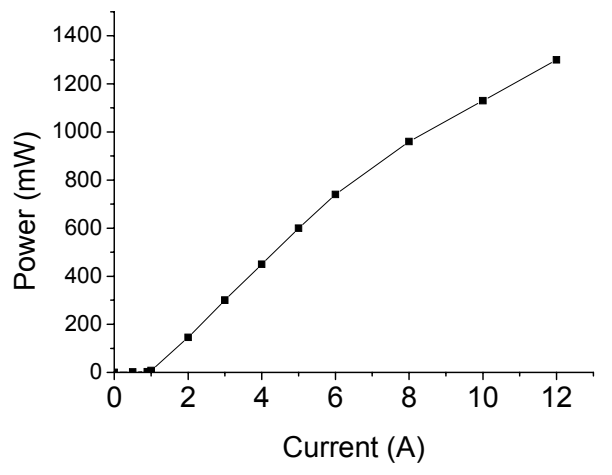

Figure 3 L-I curve without feedback

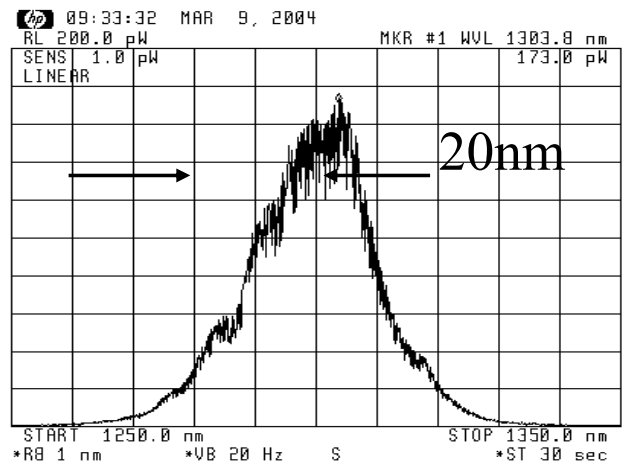

Figure 4 Emission Spectrum at 6 Amp

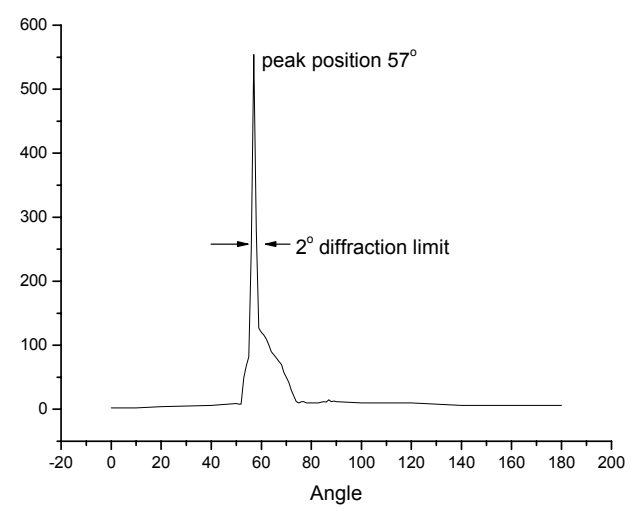

Figure 5 Far Field at 6 Amp 


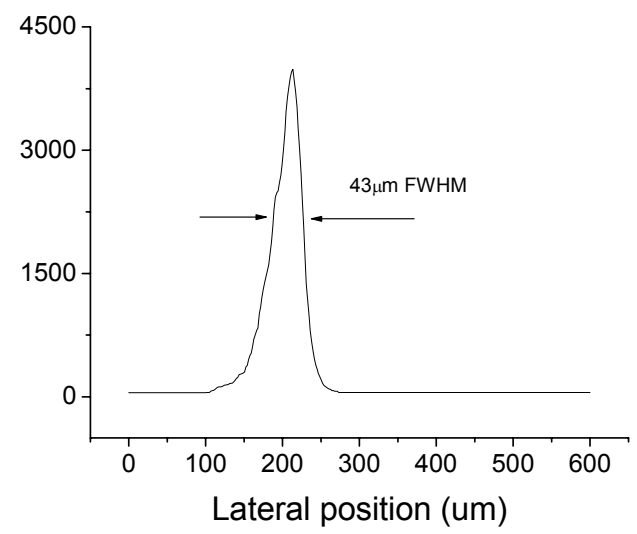

Figure 6 Near Field at 6 Amp

\section{EXTERNAL CAVITY SETUP}

Although the device by itself does not oscillate and has a broad emission spectrum, its emission wavelength can be controlled using an grating placed outside of the device. Figure 7 shows the setup of the external feedback. Two collimators are used to collect the output lights from both cleaved facets. The collimators have a focal length of $3 \mathrm{~mm}$. At one side, the collimated light is delivered to a grating that is placed in a Littrow condition. Thus the grating reflects a certain wavelength of light back to the waveguide of the device. for delivery to a grating. The grating has 600 grooves per $\mathrm{mm}$. At another side of the device, the light is focused to a photodiode for L-I measurement and then a multi-mode fiber for spectrum measurement. Near field and far field with the external feedback are again measured. The collimator is also used to image the nearfield distribution onto the slit, so the near-field pattern can be measured. For the measurement of the farfield distribution, the collimator at the right-hand side is removed.

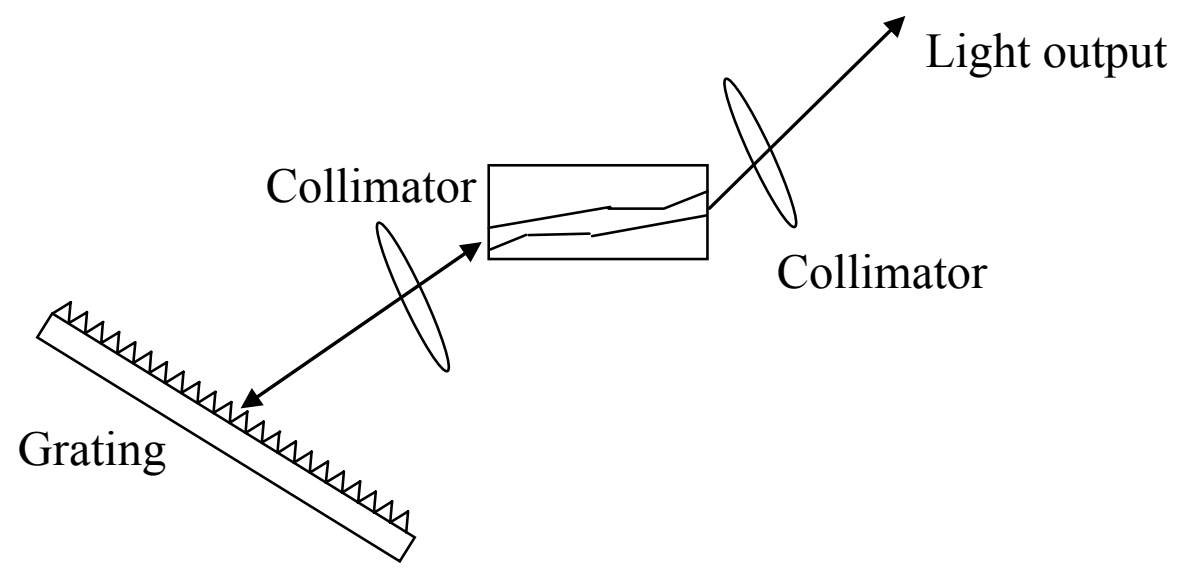

Figure. 7 Experimental setup of grating-tuned external cavity 


\section{PERFORMANCE WITH EXTERNAL FEEDBACK}

Using a grating as the feedback mirror, the emission wavelength is controlled by the grating. Then the emission wavelength becomes tunable. Figure 8 shows the tuning spectra of the modified angled broad-area semiconductor light source. With external feedback, the broadband emission with 20nm FWHM is suppressed. The output power is concentrated at the wavelength controlled by the grating feedback. The emission wavelength is tunable from $1275 \mathrm{~nm}$ to $1310 \mathrm{~nm}$. The tunable bandwidth is over $35 \mathrm{~nm}$ span and centered at $1295 \mathrm{~nm}$.
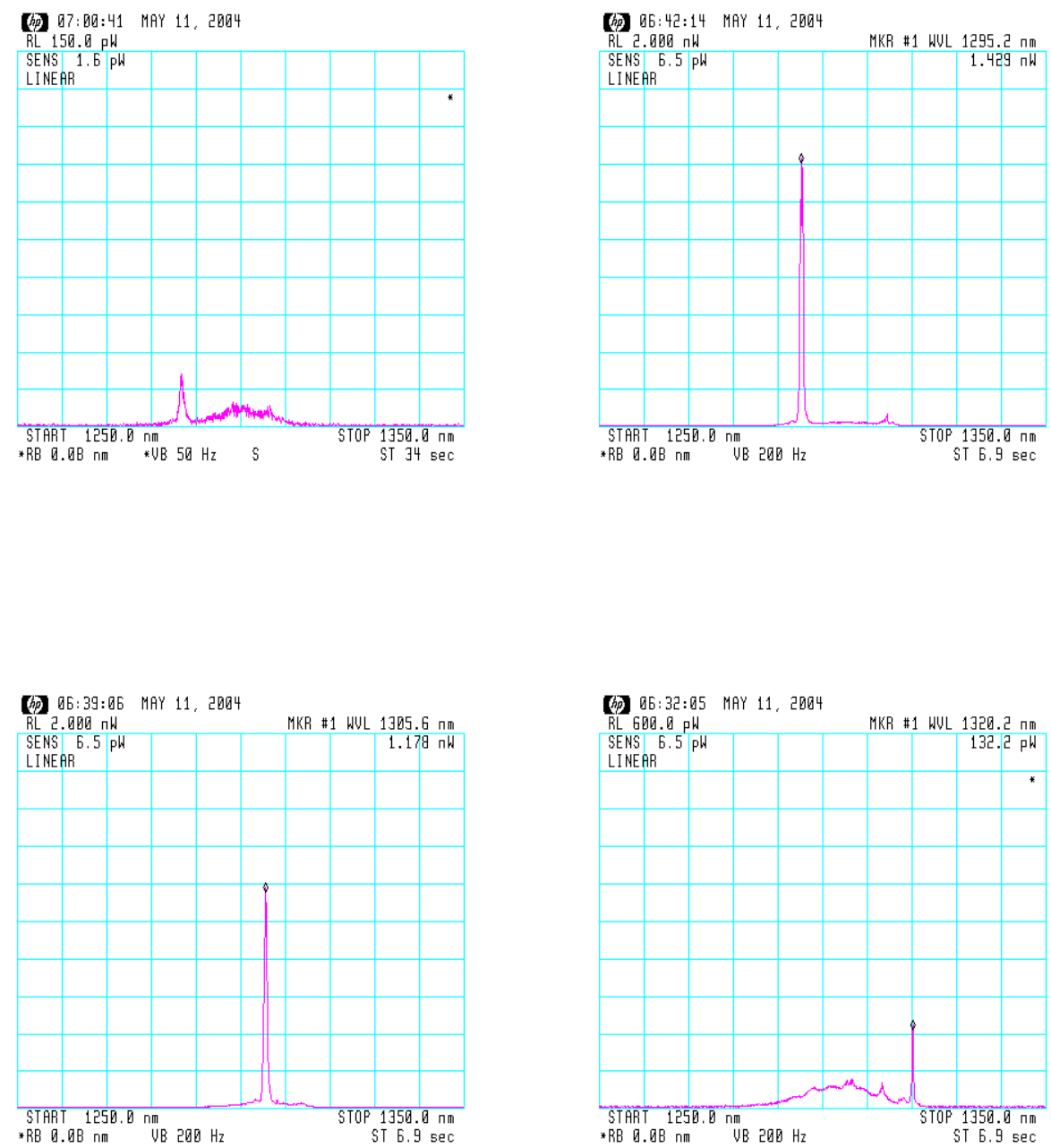

Figure 8 Tuning spectra of the modified angled waveguide 
Figure 9 shows the recorded output power versus the feedback wavelength. Figure 10 shows the recorded threshold current versus the feedback wavelength. With the external feedback, the maximum output power is $1060 \mathrm{~mW}$ at $1295 \mathrm{~nm}$ and the minimum output power is $890 \mathrm{~mW}$ at $1285 \mathrm{~nm}$ under the 6Amp of injection current. When the injection current increases, the output power also increases within the same tuning wavelength. Thus higher output power is still possible with the injection current larger than $6 \mathrm{Amp}$. In the external cavity, the lowest threshold current is $0.85 \mathrm{Amp}$ when the feedback wavelength is $1295 \mathrm{~nm}$. When grating feedback is at $1285 \mathrm{~nm}$, the threshold current is 1Amp. Figure 11 is the L-I curve with $1295 \mathrm{~nm}$ feedback. The differential efficiency is up to $0.2 \mathrm{~W} / \mathrm{A}$. The emission power can achieve $1.4 \mathrm{Watt}$ as the pumping current is $8 \mathrm{Amp}$. Figure 12 and Figure 13 show the near field and far field patterns of this device at 6Amp with grating feedback at $1295 \mathrm{~nm}$, respectively. With feedback, the near field broadens and has $48 \mu \mathrm{m}$ FWHM. The peak on far field pattern still has $2^{\circ} \mathrm{FWHM}$. The position of this peak is not affected by feedback and is still at $32^{\circ}$ away from the facet normal.

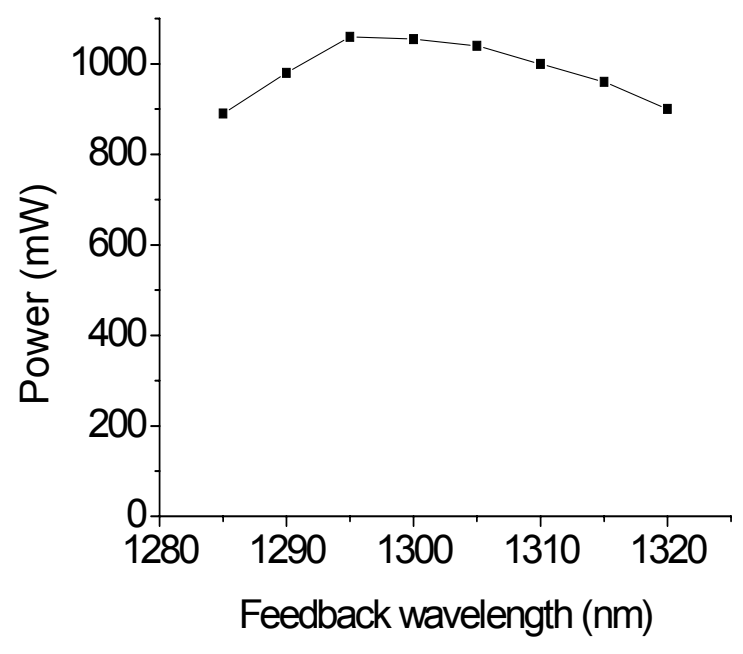

Figure 9 Power vs. Feedback wavelength at $6 \mathrm{Amp}$

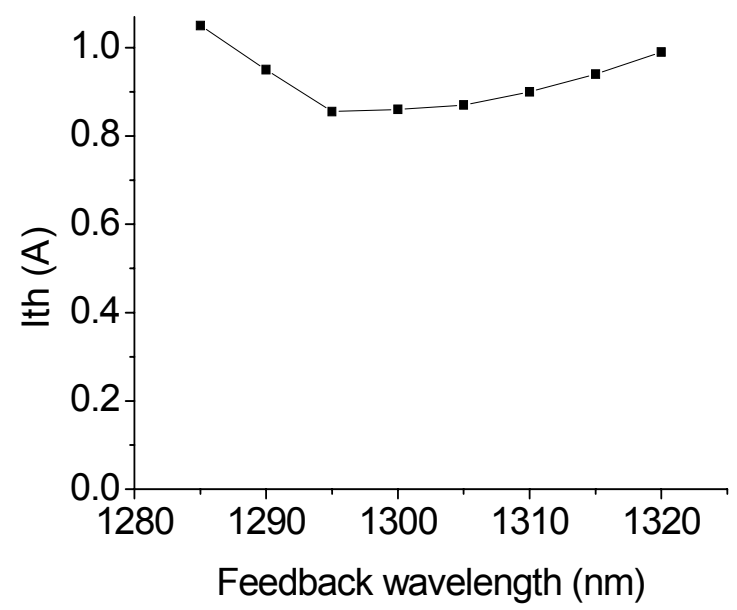

Figure 10 Ith vs. Feedback wavelength 


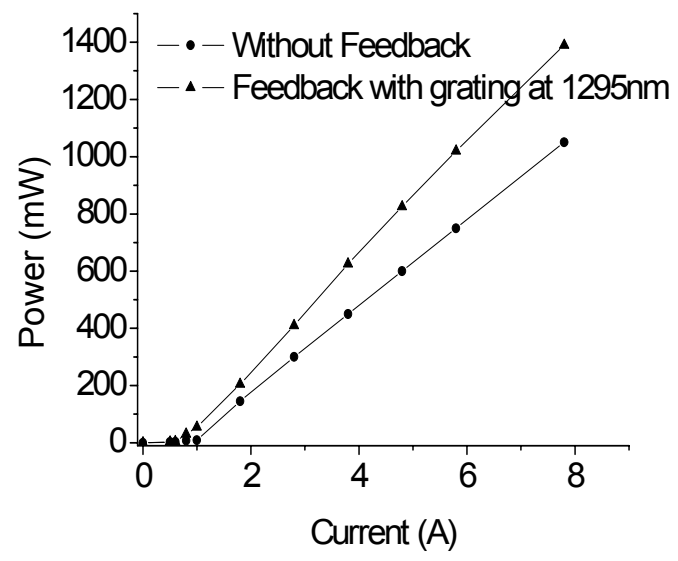

Figure 11 L-I curve with grating feedback

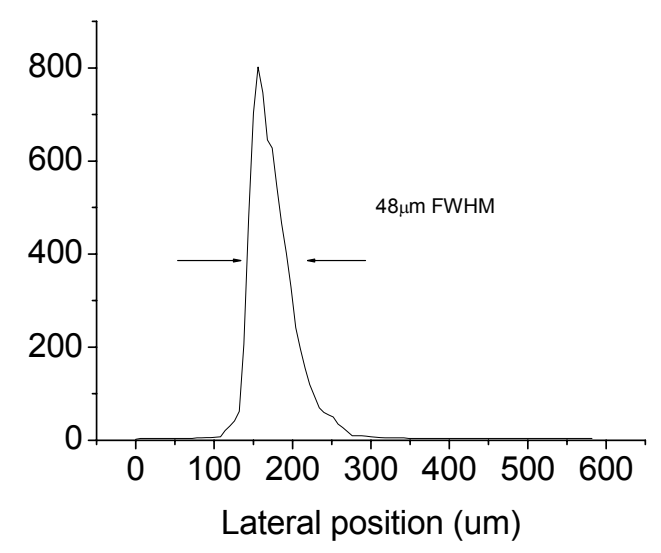

Figure 12 Near field with grating feedback.

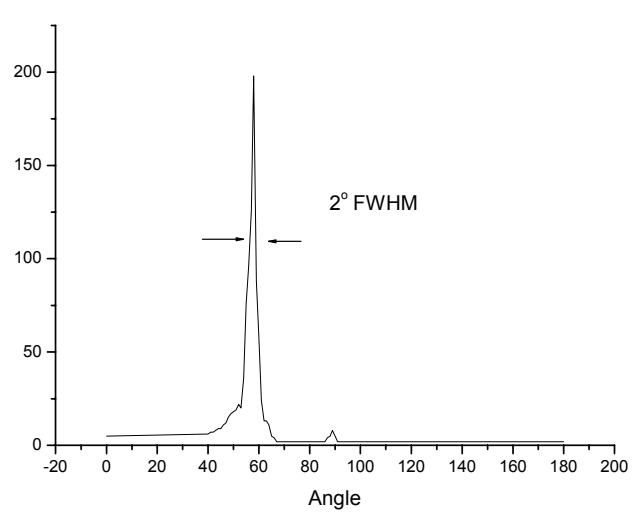

Figure 13 Far field with grating feedback. 


\section{CONCLUSION}

In conclusion, a new-type high power wavelength tunable ASE light sources are developed on a substrate with MQWs. The device length is $1960 \mu \mathrm{m}$. The waveguide is $100 \mu \mathrm{m}$ wide and tilted at $6^{\circ}$ from the facet normal. Without external feedback, this device can provide 1.4Watt ASE light per facet with $12 \mathrm{~mA}$ injection current. The emitted spectrum is broad with 20nm FWHM. The farfield has narrow divergence angle and the near field is smoothly bell-shaped without filamentation. When the external feedback is applied, the slope efficiency is increased from $0.15 \mathrm{~W} / \mathrm{Amp}$ to $0.2 \mathrm{~W} / \mathrm{Amp}$. Only $6 \mathrm{Amp}$ injection current is required to reach $1.06 \mathrm{Watt}$ output. Feedback from a grating can be used to control the emission wavelength. The nearfield pattern is slightly broadened due to external feedback while the far field pattern remains unchanged.

\section{Acknowledgement}

The authors wish to acknowledge support of National Science Council under contract number NSC 94-2112-M-002-009.

\section{REFERENCES}

1. B. Beier, D. Woll, K.-J. Boller, and R. Wallenstein, "Second-harmonic generation of the output of an AlGaAs diode oscillator-amplifier system in critically phase matched LiB3O5 and b-BaB2O4," Appl. Phys. Lett., vol. 71, pp.1-3, 1997.

2. S. G. Lambert, W. L. Casey, "Laser Communication in Space," Boston, MA: Artech, 1995.

3. P. Loosen, H.-G. Treusch, C. R. Haas, U. Gardenier, M. Weck, V. Sinnho, St. Kasperowsky, and R. vor dem Esche, "High-power diode lasers and their direct industrial applications," SPIE Proc.2382, pp.75-78, 1995.

4. M. Fukuda, "Reliability and degradation of semiconductor lasers and LEDs", Boston, MA: Artech, 1991. 5. M. A. Emanuel, N. W. Carlson, "High-efficiency AlGaAs-based laser at $808 \mathrm{~nm}$ with large transverse spot size," IEEE Photon. Technol. Lett., vol. 8, pp.1291-1293, 1996.

6. Jie-Wei Lai and Ching-Fuh Lin, "Carrier diffusion effect in flared semiconductor-laser amplifier," IEEE J. Quantum Electron. 34, pp.1247-1256, 1998.

7. J. N. Walpole, "Semiconductor amplifiers and lasers with tapered gain regions," Opt. Quant. Electron, vol.28, pp.623-645, 1996.

8. M. Mikulla, "Improved beam quality for high-power tapered diode lasers with LMG eptitaxial layer structures," SPIE Proc.3284, pp.72-79, 1998.

9. Chih-Hung Tsai, Yi-Shin Su, and Ching-Fuh Lin, "Superluminescent diodes with output power over $1 \mathrm{~W}$ and with diffraction-limited beam quality," CLEO/Europe-EQEC, Munich, Germany, Paper CE7-5-THU, 2005. 